\title{
Cielntifífica
}

\section{Identidad ambiental: múltiples perspectivas}

\author{
Environmental Identity: Multiple Perspectives
}

\section{Identidade Ambiental: Múltiplas Perspectivas}

\author{
Yair Alexander Porras Contreras ${ }^{1}$ \\ María Rocío Pérez Mesa²
}

Recibido: octubre de 2018

Aceptado: diciembre de 2018

Para citar este artículo: Porras-Contreras, Y. A., y Pérez-Mesa, M. R. (2019). Identidad ambiental: múltiples perspectivas. Revista Científica, 34(1), 123-138. Doi: https://doi.org/10.14483/23448350.14003

\section{Resumen}

El propósito del presente estudio consiste en caracterizar el material bibliográfico que se ha generado en el escenario mundial sobre la identidad ambiental. Como delimitación temporal se tomaron los últimos 18 años de producción académica presente en bases de datos de alto impacto, a partir del mapeamiento informacional bibliográfico. Para cumplir con este objetivo, se parte de la formulación de dos preguntas de investigación: ¿cuál es la producción científica (artículos) relacionada con investigaciones sobre identidad ambiental?, ¿a qué campos o áreas pertenecen las investigaciones sobre identidad ambiental y qué categorías emergen? Los resultados muestran que más del $50 \%$ de la literatura sobre identidad ambiental se concentra en dos países (Estados Unidos y Reino Unido) y que en el análisis emergen ocho campos o áreas de conocimiento en los que la identidad ambiental supone un sentido de conexión con la naturaleza, lo cual involucra habilidades, actitudes y valores que emergen en la relación con los otros.

Palabras clave: ambiente, formación ambiental, identidad ambiental, mapeamiento informacional.

\begin{abstract}
The purpose of this study is to characterize the literature that is being built on the world stage on environmental identity, taking as a timeline the academic production of the last 18 years in high impact databases. To fulfill this objective, we start with the formulation of two research questions: What is the scientific production (articles) related to research on environmental identity? What fields or areas have addressed research on environmental identity and what categories emerge? The results show that more than $50 \%$ of the literature on environmental identity is concentrated in two countries (the United States and the United Kingdom) and that in the analysis there are eight fields and/or areas of knowledge where environmental identity implies a sense of connection with nature, which involves skills, attitudes and values that emerge in the relationship with others.
\end{abstract}

Keywords: Environmental Identity, Environment, Environmental Training, Information Mapping. 


\section{Resumo}

O objetivo deste estudo é caracterizar a literatura que está sendo construída no cenário mundial sobre identidade ambiental, tomando como linha do tempo a produção acadêmica dos últimos 18 anos em bases de dados de alto impacto. Para cumprir esse objetivo, começamos com a formulação de duas questões de pesquisa: Qual é a produção científica (artigos) relacionada à pesquisa sobre identidade ambiental? Quais campos ou áreas abordaram a pesquisa sobre identidade ambiental e quais categorias emergem? ? Os resultados mostram que mais de $50 \%$ da literatura sobre identidade ambiental está concentrada em dois países (Estados Unidos e Reino Unido) e que na análise existem oito campos e / ou áreas de conhecimento onde a identidade ambiental implica um senso de conexão com a natureza, que envolve habilidades, atitudes e valores que emergem no relacionamento com os outros.

Palavras-chaves: Identidade Ambiental, Meio Ambiente, Formação Ambiental, Mapeamento de Informações.

\section{Introducción}

La producción académica y bibliográfica sobre identidad ambiental nos permite entender a esta como un concepto que cambia, se adapta y modifica en el tiempo. Siguiendo las ideas de Varela (1996), se considera la identidad ambiental como un yo emergente que se sitúa en un espacio-tiempo determinado y se consolida en un proceso autónomo, caracterizado por la construcción de significados y argumentos (Plantin, 2014). La identidad ambiental se constituye en una práctica social pluri-paradigmática (Pérez y Porras, 2005), la cual supone una diversidad de perspectivas epistemológicas, metodológicas y ontológicas con las cuales entender la complejidad ambiental. De hecho, las primeras caracterizaciones de la identidad ambiental a través de pruebas psicométricas con base en la teoría de Holland (1997) demuestran el éxito diferencial de los entornos académicos que refuerzan y recompensan patrones de habilidades e intereses asociados con una identidad fuerte, en contraste con aquellos difusos que no tienen objetivos coherentes y explícitos (Smart y Thompson, 2001).

\section{Revisión teórica del concepto identidad ambiental}

Como protagonistas de una época particularmente crucial para la historia de la humanidad, donde los cimientos del conocimiento se abren a nuevas formas de construcción y deconstrucción de la realidad, consideramos importante adelantar un análisis de aquellos aspectos relevantes que permiten un nuevo reencantamiento del mundo (Noguera, 2004). Esto para entender, en lo posible, esa mutación histórica que ha ocasionado la transición modernidad-modernidad tardía-posmodernidad, con la cual pasamos de una escisión entre el hombre y la naturaleza, el anhelo por la revolución, el progreso y la universalidad que motiva las acciones sociales e individuales (Lipovetsky, 2000) a un fracaso de la ecologización del mundo (Aledo, 2004), al aumento del individualismo hedonista (Lipovetsky, 2000) y a la búsqueda de la propia identidad (Galanés, 2006).

Desde la visión de la modernidad tardía, la identidad emerge como una construcción relacionada con la manera en que las personas y los grupos sociales se definen a sí mismos. En consonancia con estas ideas, Berger y Luckmann (2012) consideran que la identidad se explicita con la manera en que los individuos se ubican en el mundo, desplegando roles y expectativas frente a sí mismos y al colectivo que pertenecen:

En los últimos años, la articulación entre la identidad y el ambiente se ha convertido en un tema de reflexión que permite aunar elementos para la configuración del campo ambiental. De hecho, al ser considerado el ambiente como un sistema dinámico y complejo, que establece relaciones rizomáticas entre sus componentes (biofísicos, sociales y culturales), es necesario reconocer las interacciones discursivas que se construyen al interior del campo ambiental para identificar la tipología de identidades que conviven en una realidad 
ambiental compleja. Desde la configuración del campo ambiental como escenario en el que emergen relaciones asimétricas de poder (Porras et al., 2014, p. 14), se destaca el papel de algunos académicos, ciertas organizaciones y determinados grupos sociales por humanizar y democratizar las reflexiones sobre la realidad ambiental. En principio, algunos autores convergen en la idea de promover una educación ambiental crítica (EAC) con la cual se trascienda aquel "optimismo trágico" (Santos, 2009) con el que se desdibuja la complejidad de la lucha por la emancipación.

En un mundo social específico, en un espacio-tiempo particular, la identidad ambiental podría asociarse con la representación social que tienen los grupos humanos sobre sí mismos y el ambiente, la cual incluye las dimensiones cognitiva, afectiva y comportamental que en conjunto influyen en la manera como las personas se desenvuelven, organizan su vida y toman decisiones frente al propio ambiente. Una identidad ecológica o ambiental:

Se refiere a todas las diferentes formas en que las personas se construyen a sí mismas en relación con la Tierra, como se manifiesta en la personalidad, los valores, las acciones y el sentido del yo. La naturaleza se vuelve un objeto de identificación. (Thomashow, 1995, p. 3)

De acuerdo con Clayton (2003), la identidad ambiental supone:

[...] un sentido de conexión con alguna parte del entorno natural no humano, basado en la historia, el apego emocional, y/o similitud, que afecta las formas en que percibimos y actuamos hacia el mundo; una creencia de que el medio ambiente es importante para nosotros y una parte importante de quiénes somos. (2003; pp. 45 y 46)

Autores como Stets y Biga conceptualizan la identidad ambiental como los significados que las personas se atribuyen a sí mismas en relación con el entorno (2003, p. 406). De ahí que la manera en que emergen los sentidos y los significados que los sujetos otorgan a sus relaciones con el ambiente determinan desde una perspectiva compleja las actitudes y el comportamiento ambiental que exhiben en un contexto determinado. La instalación de microidentidades o ciertas predisposiciones para la acción que emergen en un determinado micromundo, es decir, aquel "espacio donde un ser humano se constituye como tal en un sistema social donde hay una recurrencia en una interacción de cooperación y se establece una autoconciencia" (Varela, 1996, p.16), confirma la idea de identidades ambientales a partir de transiciones recurrentes entre micromundos que hacen parte del campo ambiental.

En esta misma vía, Martínez-Ponce considera que la identidad ambiental es "un proceso autoreflexivo en el que los sujetos construyen, a partir de la definición de tiempo-espacio, un concepto del yo, articulado en un nosotros, y en donde el ambiente es objetivado y patrimonializado, de manera simbólica, en base a la construcción de marcos de actuación (diagnóstico, pronóstico y acción)" (2015, p. 75). Este mismo autor señala que la identidad ambiental se configura en torno a una problemática ambiental determinada, asociando el concepto de riesgo de Beck (1998) con la elaboración de construcciones colectivas frente a su resolución.

En consonancia con estas ideas, Clayton y Opotow (2003) promueven una tesis en la que se realza la interacción entre la identidad y la justicia, afirmando que es necesario adelantar estudios para comprender la complejidad de la identidad en contextos de significación específicos, con el fin de reconocer cuándo, por qué y cuán profundo se relaciona esta con los modelos de justicia que adoptan las personas. Debido a que la identidad individual y la identidad grupal permanecen imbricadas, es necesario reconocer que "una identidad grupal surge de un sentido de pertenencia, apego o participación con un grupo, basada en valores compartidos, motivaciones, características 
o experiencias que pueden convertirse en forraje para la política de identidad" (Clayton y Opotow, 2003, p. 299). Esto sin duda nos convida a pensar en la importancia del estudio de las representaciones sociales con el objeto de caracterizar la identidad individual y grupal. De hecho, algunos de estos elementos que caracterizan la identidad ambiental suponen una reflexión del individuo sobre sí mismo, el mundo material y la realidad ambiental. De igual forma, la construcción socio-simbólica de la identidad ambiental parte de una visión de mundo que elabora el individuo y el grupo social, en la cual sus creencias, concepciones, posturas epistemológicas, ontológicas, pedagógicas, éticas y políticas se traducen en prácticas discursivas y acciones propias del campo ambiental.

\section{Metodología}

Esta investigación busca configurar categorías de análisis respecto del abordaje de la identidad ambiental y su aproximación en procesos educativos mediante revisiones documentales que, además, posibiliten proyectar agendas de investigación acordes con la relevancia de la formación ambiental ciudadana para las actuales y futuras generaciones, en un país con importantes potencialidades ambientales y problemáticas que requieren ser consideradas a nivel educativo, sin que sea exclusivo del ámbito escolar. El estudio se basó en algunas de las orientaciones presentadas por Molina et al. (2012) sobre mapeamiento informacional bibliográfico (MIB), selección de fuentes bibliográficas y desarrollos conceptuales en artículos del ámbito de estudio. De igual manera, se tomó como referente para el análisis documental a Quintana y Montgomery (2006), quienes posibilitan una forma de organización del texto y representan un importante referente para adelantar la lectura crítica de los desarrollos investigativos en el escenario internacional. Para la revisión de artículos científicos se consultaron las bases de datos Science Direct, Dialnet, Eric, ProQuest, Redalyc, Scielo y Springer, bajo la orientación de las siguientes preguntas de investigación: ¿cuál es la producción científica (artículos) relacionada con investigaciones sobre identidad ambiental?, ¿a qué campos o áreas pertenecen las investigaciones sobre identidad ambiental y qué categorías emergen?

Se adelantó una revisión de más de 120 artículos, de los cuales se hizo una clasificación inicial, sistematizando aquellos pertinentes para la investigación con los siguientes datos: título del artículo, autor, revista, país, año de publicación, resumen, resultados y conclusiones. Esto permitió la reclasificación de 60 artículos, teniendo en cuenta que estos se enfocaran tanto en el estudio de la identidad ambiental como a su aproximación a nivel educativo, dando prelación a los artículos de investigación. Al respecto, emergen las siguientes categorías: concepciones, pérdida de la biodiversidad, educación ambiental, educación para el desarrollo sostenible, estrategias enseñanza-aprendizaje, reconexión y diversidad biocultural. Los datos fueron sistematizados en una hoja de cálculo de Excel con la opción filtro y se registraron en tablas; los resultados obtenidos fueron graficados y analizados para su posterior discusión con base en las preguntas formuladas.

\section{Resultados y discusión}

Para la selección de las fuentes documentales en las bases de datos se utilizaron marcadores lingüísticos, palabras claves, lectura de los resúmenes, resultados y conclusiones. Se clasificaron y reclasificaron 60 artículos publicados durante los años 2001 a 2018. En los análisis de los artículos se encontraron los siguientes resultados, respecto a las preguntas de investigación:

\section{¿Cuál es la producción científica (artículos) re- lacionada con investigaciones sobre identidad ambiental?}

La producción científica de investigaciones sobre identidad ambiental, aparece desde los albores del siglo XXI. Así, entre los años 2001 a 2005 existe 
un $5 \%$ de producción académica, al ser el período inicial en el que incursiona la temática a nivel internacional. Para el período de 2006-2010 se observa un incremento del $18,33 \%$ en las producciones; para el período de 2011-2015 un aumento del 41,66 \% en las publicaciones; seguido del período 2015-2018, con un $35 \%$ de trabajos de investigación, lo cual muestra un importante incremento durante el último lustro, como se observa en la figura 1.

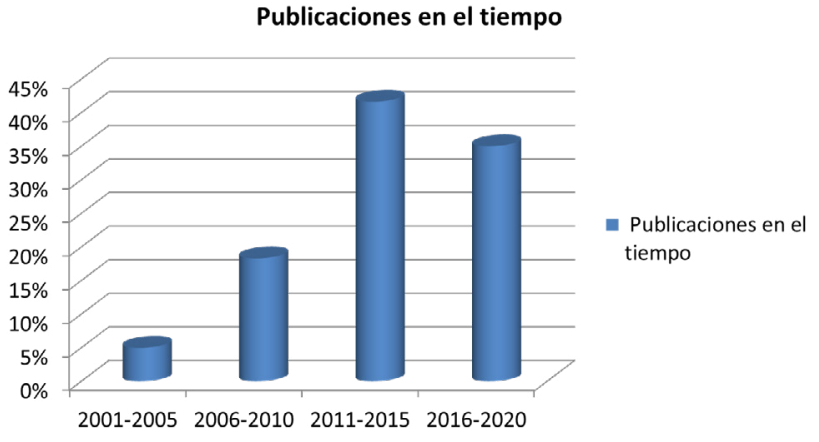

Figura 1. Publicaciones por periodos de tiempo.

Fuente: elaboración propia.
Como se aprecia en la figura 2, la producción investigativa sobre la identidad ambiental por países permite identificar aquellos que cuentan con una destacada producción en este ámbito, como es el caso de Estados Unidos con un $35 \%$, seguido de Reino Unido con un $17 \%$ y España con un $10 \%$. Otros países como Australia, que cuentan con el $8 \%$, y Holanda, con el $7 \%$, se encuentran avanzando en esta materia. En menor proporción aparecen países como Italia, Francia, Israel y China, cada uno con un 3 \%; mientras que Suecia, Portugal, Polonia, Brasil, Colombia, Israel y Malasia aparecen con un $2 \%$, respectivamente.

Si se considera el reporte de las publicaciones y su distribución por continentes, se encuentra que el continente europeo tiene una destacada producción, empezando por el Reino Unido, seguido de España, Holanda, Francia e Italia, y en menor proporción Portugal y Polonia, que en conjunto representan el $45 \%$. El segundo continente con la mayor producción corresponde a América, en el

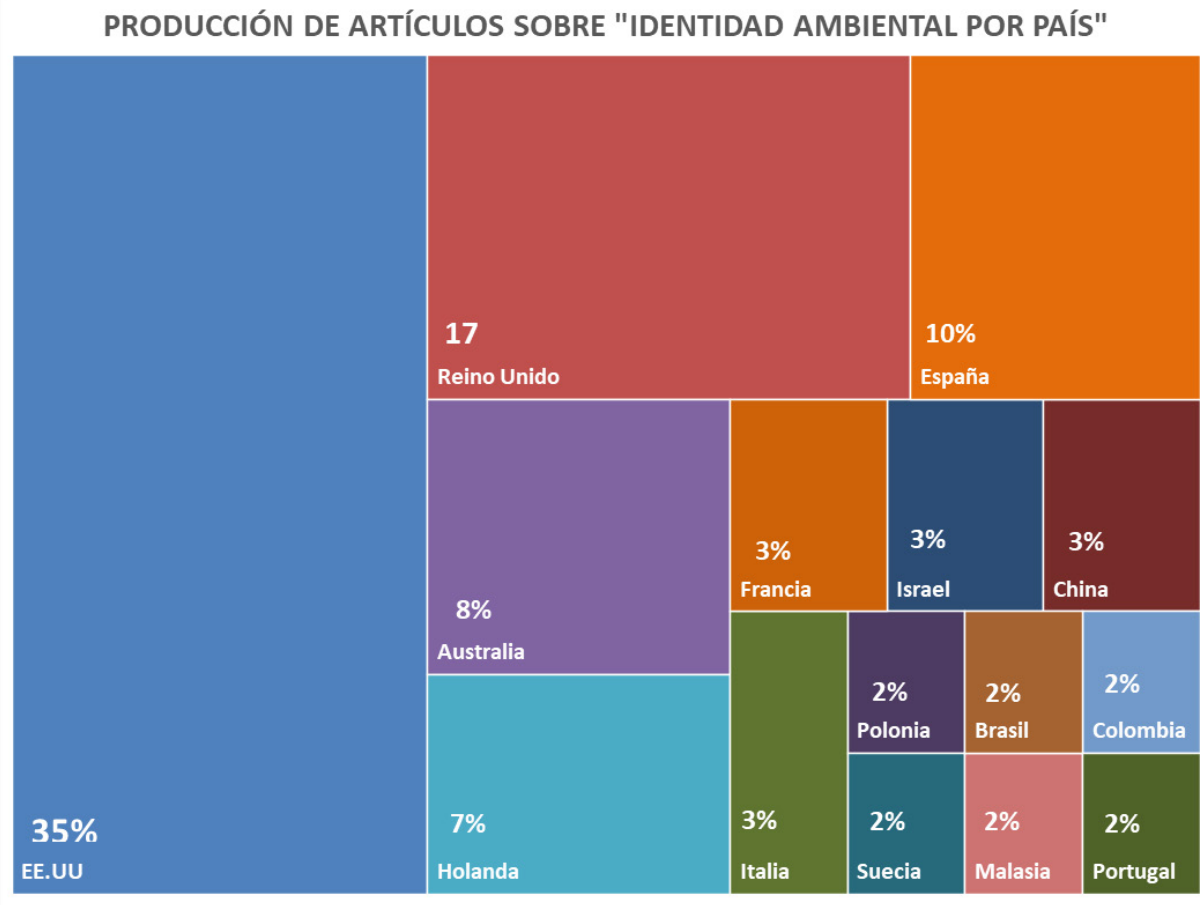

Figura 2. Producción de artículos sobre Identidad Ambiental.

Fuente: elaboración propia. 
cual se destaca que el mayor porcentaje se sitúa en Estados Unidos, mientras que países como Brasil y Colombia tienen una menor proporción de la producción que en conjunto corresponde al 38,33\%. En tercer y cuarto lugar, con el mismo porcentaje de producción, se encuentran Asia con el 8,33\% y Oceanía con el 8,33\%, mientras que África no presenta producción a este respecto (figura 3).

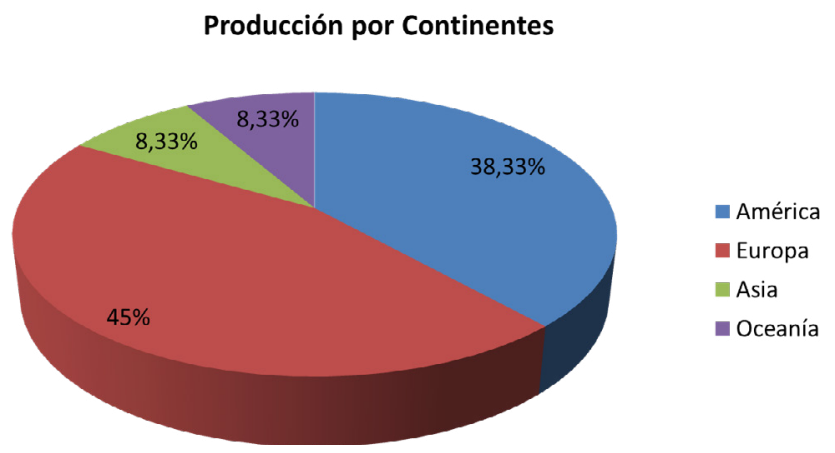

Figura 3. Producción de artículos sobre identidad ambiental por continentes.

Fuente: elaboración propia.

En esta revisión es posible establecer que la producción de investigación sobre identidad ambiental se empieza a visibilizar ampliamente durante los inicios del siglo XXI y, especialmente, durante la última década, lapso en el que se concentra un mayor porcentaje de publicaciones. Además, se logra identificar que la producción por países se concentra en Estados Unidos y Reino Unido (52 $\%$, aspectos que muestran la relevancia del tema y su potencialidad como objeto de investigación en América, Europa, Asia y Oceanía.

\section{¿A qué campos o áreas pertenecen las investiga- ciones sobre identidad ambiental y qué categorías emergen?}

De acuerdo con el análisis de los resúmenes, los resultados y las conclusiones de los artículos, es posible identificar ocho campos o áreas de investigación (figura 4). Cuatro de las cuales se han consolidado en la literatura como son: psicología ambiental, educación ambiental, sociología ambiental y economía ambiental. Los restantes cuatro campos o áreas corresponden a ámbitos emergentes que vienen incursionando en las discusiones sobre la configuración de la identidad ambiental en escenarios diversos. Estos campos emergentes son administración ambiental, geografía y ambiente, estudios culturales y educación en ciencias y comunicación e imagen ambiental corporativa.

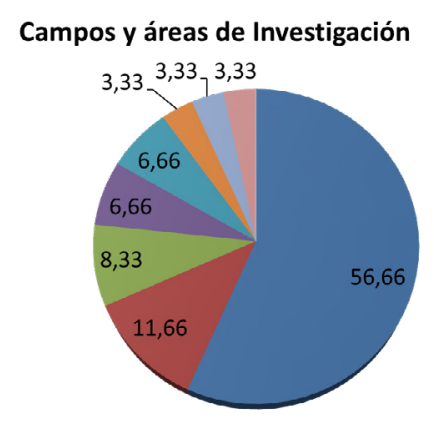

$$
\begin{aligned}
& \text { - Psicología Ambiental } \\
& \text { - Educación Ambiental } \\
& \text { Sociología Ambiental } \\
& \text { - Economía Ambiental } \\
& \text { administración Ambiental } \\
& \text { Geografía y Ambiente } \\
& \text { Estudios culturales y Ed. } \\
& \text { Ciencias } \\
& \text { Comunicación e imagen } \\
& \text { ambiental corporativa }
\end{aligned}
$$

Figura 4. Campos y áreas de investigación.

Fuente: elaboración propia.

\section{Campos o áreas de investigación asociados con la configuración de la identidad ambiental en la literatura}

\section{Psicología ambiental}

La literatura asociada a la construcción de la identidad en el campo de la psicología ambiental revela una tendencia mayoritaria en la producción académica analizada (5,66 \%), lo cual permite reconocer que uno de los temas de estudio significativo para un número considerable de investigadores es la interacción entre los seres humanos y el medio en el que se desenvuelven (Hinds y Sparks, 2008; Winter y Chavez, 2008; Hinds y Sparks, 2009). Si bien existen variables psicológicas y conductuales que se asocian a la consolidación de la relación entre el hombre y la naturaleza, es claro que la complejidad de esta conexión supone la adopción de principios epistemológicos, ontológicos y axiológicos que proveen un componente reflexivo a la discusión sobre la identidad ambiental. 
La importancia de consolidar positivamente los procesos de construcción de la identidad ambiental a partir de la fuerza de conexión afectiva con el entorno natural se convierten en derroteros de la psicología ambiental (Devine-Wright y Clayton, 2010; Swim et al., 2014; Kashima, Paladino y Margetts, 2014). Algunos estudios revelan la importancia de evaluar ciertos componentes de la identidad ambiental a partir de escalas actitudinales y cuestionarios (Olivos y Aragonés, 2011; Olivos et al., 2014; Navarro, Olivos y Fleury-Bahi, 2017), mientras que otros refrendan la identificación psicológica de las personas con el entorno y la predisposición para adelantar iniciativas ambientales como la exposición, la familiarización y el apego por la naturaleza, con base en la identidad ecológica y el esfuerzo individual (Morton, Van der Bles y Haslam, 2017; Rodríguez y Ortega, 2017).

La interacción entre el apego de lugar y la identidad ambiental, con base en la apropiación, la satisfacción residencial, la recuperación del entorno, las actitudes ambientales y los comportamientos proambientales (Hernández et al., 2010; Marcouyeux y Fleury-Bahi, 2011;), se convierte en una temática común para la construcción de relaciones más estrechas entre los integrantes de la comunidad. A su vez, la memoria de lugar asociada al significado y a la identidad del lugar (Lewicka, 2008) brinda elementos que permiten reconocer la percepción que los residentes tienen del vecindario, lo cual genera nuevas relaciones grupales que determinan una identidad colectiva (Casakin, Hernández y Ruiz, 2015). Por ejemplo, la percepción del riesgo asociada a factores psicológicos que incluyen la preocupación, el apego al lugar y la identidad ambiental constituyen aspectos prioritarios para la organización colectiva y la toma fundamentada de decisiones (Ujang y Zakariya, 2015; Pavlova y Silbereisen, 2015; Bernardo y Palma-Oliveira, 2016; Shaolian, 2017; Spence, Pidgeon y Pearson, 2018).

Diferentes investigaciones han centrado su foco de estudio en la relación entre la identidad ambiental y la participación de las personas en actividades ecológicas (Van der Werff, Steg y Keizer, 2013, 2014; Dono, Webb y Richardson, 2010), reconociendo que la autoidentidad ambiental depende de los valores biosféricos y del comportamiento pasado, lo cual sugiere que el comportamiento respetuoso con el ambiente se puede promover a partir del activismo ambiental, la evocación de acciones pasadas con consecuencias futuras y la inclusión de la moral ambiental a partir de la preocupación por las especies humanas y no humanas (Jia et al., 2017), prestando especial atención al cuidado por el ambiente, la insatisfacción ante comportamientos irresponsables y la reflexión sobre la justicia ambiental (Clayton y Opotow, 2003).

El tema del cuidado de los recursos no es ajeno a las discusiones sobre la construcción de la identidad ambiental. De hecho, la promoción de comportamientos favorables con el ambiente a través del aprendizaje de iniciativas "catalizadoras" (Whitmarsh y O'Neill, 2010) se posiciona como una alternativa para combatir la resistencia al cambio (Murtagh, Gatersleben, y Uzzell, 2012) y particularmente la internalización de una identidad propia de ahorro (Mallett y Melchiori, 2016). Consecuentemente, el debate sobre la configuración de la identidad ambiental a partir de la teoría del comportamiento planificado (Fielding, McDonald y Louis, 2008; Carfora, Caso, Sparks y Conner, 2017) se centra en la validación de un modelo para predecir las intenciones de las personas en una situación ambiental. En este sentido, la consolidación de la identidad ambiental, junto con beneficios económicos y ambientales percibidos después de participar en actividades ambientales (Li, Kim, Lee y Griffin, 2016), desempeña un papel importante en el grado de satisfacción que evidencian los residentes de una zona, específicamente cuando se participa en un programa de "aprendizaje ambiental" (Jaksha y Johnson, 2013).

\section{Educación ambiental}

El campo de la educación ambiental en su acontecer histórico ha desarrollado diferentes líneas de 
investigación y de manera reciente ha empezado a estudiar la identidad ambiental en contextos educativos, explorando sus relaciones con la identidad de lugar y su interacción con comportamientos ambientalmente responsables a nivel universitario, demostrando la importancia de los procesos educativos que contribuyen a fortalecer la identidad del lugar y la responsabilidad ambiental en la vida cotidiana (Lawrence, 2012; Sola, 2014), así como la conexión entre las identidades de consumo y las problemáticas ambientales (Blatt, 2014).

De otra parte, algunas investigaciones se ocupan de explorar procesos de enseñanza y aprendizaje en los contextos escolares asociados con las identidades ambientales, advirtiendo el papel del aprendizaje dialógico en el contexto de la educación ambiental, señalando que los individuos no tienen una "jerarquía de identidad" sino un self dialógico que atribuye sentido a las experiencias tanto de manera consciente como inconsciente (Meijers, Lengelle y Kopnina, 2016).

En otra investigación se exploran los procesos de construcción de la identidad ambiental a través de la indagación sobre cómo los niños de quinto grado ven su relación con el mundo natural. Se encontró que los niños reconocen y describen su propia identidad ambiental, pero esa identidad a menudo no se considera en el aula de ciencias, por lo cual se concluye que esta falta de reconocimiento puede limitar las conexiones de la ciencia escolar con los intereses de los niños y el apego emocional al mundo natural (Tugurian y Carrier, 2017).

Otra línea de trabajo corresponde a la indagación de la identidad ambiental en grupos que tienen un vínculo con lo ambiental. Para el caso de los grupos ambientales se propone comprender el rango de actitudes y comportamientos asociados con el trabajo voluntario, destacándose un mayor grado de atención a los problemas ambientales, la identidad ambiental y los comportamientos proambientales, además de ser más propensos a sentirse apegados a su entorno local (Dresner, Handelman, Braun y Rollwagen-Bollens, 2015).
Otro de los grupos corresponde a una comisión de adolescentes de diversos lugares del mundo, quienes participaron en un programa de educación global, relacionado con el cambio climático en Asia. En este grupo se aprecia la importancia de la teoría de la identidad sociocultural para explorar cómo la práctica, la acción y el reconocimiento pueden facilitar el desarrollo de la identidad ambiental a través de procesos de interacción con personas afectadas por el cambio climático (Stapleton, 2015).

\section{Sociología ambiental}

El campo de la sociología ambiental plantea un trabajo importante, en relación con la identidad ambiental, al problematizar desde diferentes perspectivas las relaciones de la sociedad con la naturaleza, especialmente al considerar el papel que tienen los grupos en la construcción social del ambiente. En tal sentido, se empiezan a desplegar diversos ejes que por una parte, abordan las relaciones entre las identidades ambientales, la agencia individual y su influencia en el comportamiento ambiental receptivo, que abogan más por una relación entre identidades y comportamientos, más que de actitudes (Stets y Biga 2003). Igualmente, se discute la identidad ambiental en relación con la identidad de lugar, lo cual busca trascender aquellas lecturas del lugar examinadas con mayor frecuencia en términos físicos, para reconocer el lugar como una construcción social que permite la construcción de una identidad específica basada en la clase (Burley et al., 2012).

También, existen otras miradas que desde el conflicto en la gestión de los recursos naturales y ambientales problematizan la identidad ambiental, desde la identidad social, lo cual aporta en la comprensión de los factores que impulsan dichos conflictos, así como en la necesidad de considerarlos una serie de episodios a través del tiempo y de los contextos, convirtiéndose en un modelo integrador del panorama sociopolítico (Robertson, 2013). 
De otra parte, se destaca un interés por enfocar investigaciones hacia el estudio de sociedades ecológicamente sustentables, en las cuales se avanza hacia la caracterización de las identidades ambientales y las acciones de grupos, como el del sector de agricultores y su importancia en el manejo de esquemas agroambientales, a propósito de los procesos de conservación (Colvin, Witt y Lacey, 2015). Así mismo, se avanza hacia propuestas de intervención con comunidades educativas para democratizar el conocimiento y fomentar habilidades que a su vez contribuyen al desarrollo de una identidad ecológica y ciertos comportamientos ambientales (Riley, 2016).

\section{Economía ambiental}

En el campo de la economía ambiental se aprecian diferentes aproximaciones de trabajos e investigaciones a la hora de abordar la identidad ambiental. En tal sentido, se encuentran desarrollos que problematizan las relaciones de los seres humanos con la naturaleza a propósito de la deuda ecológica, el acaparamiento de tierras, entre otros; los cuales apelan a la justicia ambiental, permitiendo elaborar conceptos de dichas realidades, de manera que se propende por una investigación comparativa para la comprensión del uso de lenguajes de valoración, diferentes del crecimiento económico "verde" o el desarrollo sostenible (Anguelovski y Martínez Alier, 2014). Otro aspecto que problematiza la relación está asociado con el enfoque de las capacidades y el de los servicios ecosistémicos, en los que se discute la capacidad de identidad personal y la ventana de aspiraciones, ambas relacionadas con los servicios de los ecosistemas culturales (Jia et al., 2017)

En este campo también se encuentran investigaciones que buscan comprender el tema del consumismo, la ética y la identidad ambiental, en los que se resaltan las realidades que actualmente se tienen y la necesidad de reconocer los valores personales, la autoidentidad verde, los motivos éticos y la apertura al cambio. En algunas investigaciones se ha hallado que los valores de conservación poco se traducen en las intenciones de adoptar automóviles eléctricos y tienden a considerar menos los aspectos ecológicos y morales en las elecciones de consumo (Barbarossa, De Pelsmacker y Moons, 2017). Por otra parte, se trabaja en la nueva conceptualización de la autoidentidad proambiental (Aipa), considerada relevante para el futuro de mercados emergentes y cuya investigación permitió establecer que esta se activaba de forma múltiple mediante pautas situacionales, responsabilidad moral, evaluación, deseabilidad social, siendo matizada por la eficacia y el conocimiento del consumidor (Ballet, Marchand, Pelenc y Vos, 2018).

\section{Administración ambiental}

En el campo de la administración y gestión ambiental se empieza a trabajar en propuestas de investigación que se enfocan especialmente en tratar la gestión de parques locales, zonas de conservación y áreas protegidas. De igual manera, se hace un especial énfasis en la gobernanza y el manejo de residuos, fortaleciendo la identidad ambiental de sus habitantes o visitantes, entendiendo las presiones del desarrollo urbano frente a escenarios naturales y la falta de atención de problemas como la contaminación por residuos. Aspectos que en general muestran una radiografía de las condiciones en que se encuentran ciertas regiones del planeta en las que se ha dado un acelerado crecimiento de las áreas urbanas que afectan de diversas maneras los sistemas ecológicos y la biodiversidad (White, Virden y Van Riper, 2008; Howell, 2017; Bleam, 2018).

A este respecto, las investigaciones exploran el efecto de la experiencia previa con el entorno y las dos dimensiones del lugar adjunto (identidad de lugar y dependencia de lugar) en visitantes de las zonas de reserva para la toma de decisiones en la gestión sustentable del lugar (White, Virden y Van Riper, 2008). También examinan lugares significativos para "administradores" (voluntarios de áreas protegidas), encontrando que estos elaboran 
significados expresivos de identidad que trascienden y atraviesan escalas geográficas, lo que permite señalar que el concepto de identidad de lugar es más que una subconstrucción de lugar adjunto y requiere una mayor teorización como una experiencia fenomenológica-encarnada (Bleam, 2018). También se expresan líneas de trabajo que plantean tensiones entre el desarrollo urbano y el mantenimiento de espacios naturales, como también el elevado número de residuos sólidos que se constituyen en foco de contaminación en las urbes. En este sentido, se investiga con trabajadores o habitantes de los lugares, la manera de fortalecer la identidad ambiental y el manejo sustentable de los lugares (Howell, 2017).

\section{Geografía y ambiente}

En lo concerniente a la geografía se encuentran trabajos relacionados con conceptos nodales como ambiente, cultura e identidad de lugar, en los cuales se exploran sus conexiones o interrupciones desde una perspectiva crítica. En estos se asume que las nociones de lugar son múltiples y fluidas, y la transformación del lugar en áreas rurales y regionales puede ser más rápida frente a la comprensión cambiante del lugar que tienen los residentes. Por tanto, el sentido de lugar resulta más importante y de largo plazo para los residentes rurales, que la misma identidad de lugar, a propósito del cambio del paisaje en las regiones, lo cual implica un proceso de identificación con las fuerzas globalizadoras del desarrollo (Carter, Dyer y Sharma, 2007).

De otra parte, el abordaje de la identidad ambiental mediante un trabajo comparado de los procesos de transformación identitaria que han ocurrido en el trapecio amazónico colombiano y en el archipiélago de San Andrés permiten considerarlos como dos casos de redefinición histórica de la etnicidad con referencia al medio geográfico, y a las tensiones que los capitales y agentes institucionales generan en torno a lo tradicional (De La Cruz, 2014).

\section{Estudios culturales y enseñanza de las ciencias}

En este ámbito de la enseñanza de las ciencias, se plantea la relevancia de implementar preguntas y problemáticas asociadas con el papel de las identidades en el abordaje de cuestiones sociocientíficas con escolares. En especial al tratar aspectos sobre las representaciones de la naturaleza, la biodiversidad y los ecologistas que permiten enriquecer los debates y aportar a procesos de formación de sus propias identidades (López-Facal y Jiménez-Aleixandre, 2009). Tam bién, desde el lugar de los profesores en formación inicial, se examina la percepción respecto a la identidad de lugar y su identidad docente, logrando a través de un curso titulado "Integrando la" que los estudiantes tengan una variedad de experiencias con las cuales resignificar aspectos de la identidad de lugar que vinculan lo familiar, la pertenencia, la implicación y el sentido (Gross y Hochberg, 2016).

\section{Responsabilidad corporativa}

En años recientes la discusión sobre la responsabilidad corporativa y los procesos de formación ambiental han cobrado especial interés, sobre todo al examinar posibles relaciones entre la identidad ambiental y la responsabilidad ambiental corporativa. En este sentido, se contempla la importancia de lo que se ha denominado "Comportamientos de ciudadanía organizacional para el medio ambiente" (CCOA) y de igual forma, la "Responsabilidad ambiental de megaproyectos" (RAM). Los resultados muestran que las percepciones de los participantes del proyecto sobre las prácticas de RAM dirigidas a los interesados internos (vinculados en contratos del proyecto) están relacionadas de manera positiva con sus CCOA. Por el contrario, las percepciones de los participantes del proyecto de las prácticas de RAM dirigidas a los interesados externos (la comunidad local y el público en general) tienen un impacto insignificante en sus CCOA (Wang et al., 2017). 
Una nueva línea de trabajo se enfoca en la necesidad de desarrollar una identidad ambiental fuerte que involucre a toda la organización. Para ello, la comunicación e imagen ambientalmente responsable es relevante. El estudio de caso, adelantado con una empresa sueca dedicada a la industria de la construcción y administración de propiedades, permitió identificar que la organización había creado una sólida identidad ambiental corporativa con visiones claras y representaciones simbólicas. Sin embargo, la identidad ambiental organizacional en la que los miembros de la organización se identifican con las actividades ambientales no se desarrolló completamente, lo que se atribuye a una comunicación descendente (Adamsson, 2012).

\section{Conclusiones}

A partir de la revisión documental sobre identidad ambiental en bases de datos de alto impacto, queda claro el carácter emergente en su consolidación teórica. Se resalta que la producción académica sobre el tema se concentra en países como Estados Unidos y el Reino Unido (52 \%), destacándose el interés ascendente en el estudio del tema en países no angloparlantes.

Un común denominador en estos trabajos es la relevancia que se le brinda a los procesos de construcción de la identidad ambiental en diferentes ámbitos de interacción social, ya que su estudio permite a las personas, además de elaborar significados y sentidos en torno al ambiente, consolidar iniciativas de interacción social, convirtiéndose en un catalizador para la comprensión y transformación de la realidad ambiental.

De acuerdo con el análisis de los artículos, es posible identificar ocho campos o áreas de investigación relacionados con la identidad ambiental (psicología ambiental, educación ambiental, sociología ambiental y economía ambiental, administración ambiental, geografía y ambiente, estudios culturales y educación en ciencias, y comunicación e imagen ambiental corporativa).
Los anteriores reivindican el carácter diverso de la realidad ambiental, pues si bien es cierto que demuestran la pluri-paradigmaticidad del campo ambiental, promueven el fortalecimiento de la confianza por la capacidad humana de crear horizontes de posibilidad. Consecuentemente, la diversidad de posturas teóricas frente a la identidad ambiental, sus interacciones con otros ámbitos del actuar humano y sus implicaciones en los procesos de formación ambiental se constituyen en posibilidades de orden ontológico y epistemológico para comprender la manera en que las personas habitan el mundo, forman su autoconcepto y reflexionan sobre su realidad social. La construcción de identidades individuales y grupales contribuye a la elaboración de discursos, acuerdos, disidencias y distanciamientos entre posturas diversas a partir de la elaboración de un sentido de conexión con la naturaleza, lo cual involucra habilidades, actitudes y valores que emergen de la configuración de la identidad ambiental a partir de patrones de interacción entre el self dialógico y la naturaleza.

\section{Referencias}

Adamsson, E. (2012). Forming and Communication of an Environmental Identity and Image. (tesis de máster). Södertörn University, Suecia.

Aledo-Tur, A. (2004). El fracaso de la ecologización del mundo: Ética y postecología. En A. Aledo, L. Galanes y J. Domínguez, Ética y ecología: la crisis ética de los movimientos ecologistas contemporáneos. San Juan de Puerto Rico: Editorial Tal Cual, Universidad de Puerto Rico.

Anguelovski, I., y Martínez Alier, J. (2014). The "Environmentalism of the Poor" revisited: Territory and place in disconnected glocal struggles. Ecological Economics, 102, 167-176. DOI: https://doi.org/10.1016/j.ecolecon.2014.04.005

Ballet, J., Marchand, L., Pelenc, J. y Vos, R. (2018). Capabilities, Identity, Aspirations and Ecosystem Services: An Integrated Framework. 
Ecological Economics, 147, 21-28. DOI: https://doi.org/10.1016/j.ecolecon.2017.12.027

Barbarossa, C., De Pelsmacker, P. y Moons, I. (2017). Personal Values, Green Self-identity and Electric Car Adoption. Ecological Economics, 140, 190-200. DOI: https://doi.org/10.1016/j.ecolecon.2017.05.015

Beck, U. (1998). La sociedad del riesgo. Hacia una nueva modernidad. Barcelona: Editorial Paidós.

Berger, P. y Luckmann, T. (2013). La construcción social de la realidad. Madrid: Amorrortu-Murguía.

Bernardo, F. y Palma-Oliveira, J. M. (2016). Urban neighbourhoods and intergroup relations: The importance of place identity. Journal of Environmental Psychology, 45, 239-251. DOI: https://doi.org/10.1016/j.jenvp.2016.01.010

Blatt, E. (2014). Uncovering Students Environmental Identity: An Exploration of Activities in an Environmental Science Course. Journal of Environmental Education, 45(3), 194-216. DOI: https://doi.org/10.1080/00958964.2014.911139

Bleam, R. M. (2018). Unbounded place meanings and embodied place identities for conservation volunteers in Scottsdale, Arizona. Journal of Environmental Psychology, 56, 76-83. DOI: https://doi.org/10.1016/j.jenvp.2018.03.002

Burley, D., Daunis, C., Walker, M., Shelton, N., Cuifi, J., Coleman, M. y Matheu, B. (2012). Imaginatio enviro-station: Students connecting students to ecological sustainability. Journal of Rural Social Sciences, 27(2), 50-71

Carfora, V., Caso, D., Sparks, P. y Conner, M. (2017). Moderating effects of pro-environmental self-identity on pro-environmental intentions and behaviour: A multi-behaviour study. Journal of Environmental Psychology, 53, 92-99. DOI: https://doi.org/10.1016/j. jenvp.2017.07.001

Carter, J., Dyer, P. y Sharma, B. (2007). Dis-placed voices: Sense of place and place-identity on the Sunshine Coast. Social and Cultural Geography, 8(5), 755-773. DOI: https://doi. org/10.1080/14649360701633345
Casakin, H., Hernández, B. y Ruiz, C. (2015). Place attachment and place identity in Israeli cities: The influence of city size. Cities, 42(PB), 224-230. DOI: https://doi.org/10.1016/j. cities.2014.07.007

Clayton, S. (2003). Environmental identity: A conceptual and an operational definition. En S. Clayton y S. Opotow (eds.), Identity and the natural environment. The psychological significance of nature (pp. 45-65). Cambridge: The MIT Press. DOI: https://doi.org/10.7551/ mitpress/3644.003.0005

Clayton, S. y Opotow, S. (2003). Justice and Identity: Changing Perspectives on What Is Fair. Personality and Social Psychology Review, 7(4), 298-310. DOI: https://doi.org/10.1207/ S15327957PSPR0704 03

Colvin, R. M., Witt, G. B. y Lacey, J. (2015). The social identity approach to understanding socio-political conflict in environmental and natural resources management. Global Environmental Change, 34, 237-246. DOI: https:// doi.org/10.1016/j.gloenvcha.2015.07.011

De La Cruz, P. (2014). Identidades ambientales, un caso comparativo entre el trapecio Amazónico y el Archipiélago de San Andrés. Cuadernos de Geografía. Revista Colombiana de Geografía, 23(1), 125-131. DOI: https://doi. org/10.15446/rcdg.v23n1.41088

Devine-Wright, P. y Clayton, S. (2010). Introduction to the special issue: Place, identity and environmental behaviour. Journal of Environmental Psychology, 30(3), 267-270. DOI: https://doi.org/10.1016/S0272-4944(10)00078-2

Dono, J., Webb, J. y Richardson, B. (2010). The relationship between environmental activism, pro-environmental behaviour and social identity. Journal of Environmental Psychology, 30(2), 178-186. DOI: https://doi.org/10.1016/j. jenvp.2009.11.006

Dresner, M., Handelman, C., Braun, S. y Rollwagen-Bollens, G. (2015). Environmental identity, pro-environmental behaviors and civic engagement of volunteer stewards in Portland 
area parks. Environmental Education Research, 21(7), 991-1010. DOI: https://doi.org/10.1080 /13504622.2014.964188

Fielding, K. S., McDonald, R. y Louis, W. R. (2008). Theory of planned behaviour, identity and intentions to engage in environmental activism. Journal of Environmental Psychology, 28(4), 318-326. DOI: https://doi.org/10.1016/j. jenvp.2008.03.003

Galanés, L. (2006). Ética ecologista para una sociedad sin moral: sobre la relación conflictiva entre el ecologismo y el liberalismo contractualista. Portularia, 4(2), 21-38.

Hernández, B., Martín, A. M., Ruiz, C. y Hidalgo, M. del C. (2010). The role of place identity and place attachment in breaking environmental protection laws. Journal of Environmental Psychology, 30(3), 281-288. DOI: https://doi.org/10.1016/j.jenvp.2010.01.009

Gross, M. y Hochberg, N. (2016). Characteristics of place identity as part of professional identity development among pre-service teachers. Cultural Studies of Science Education, 11(4), 1243-1268. DOI: https://doi.org/10.1007/ s11422-014-9646-4

Hinds, J y Sparks, P. (2008). Engaging with the natural environment: The role of affective connection and identity. Journal of Environmental Psychology, 28(2), 109-120. DOI: https://doi. org/10.1016/j.jenvp.2007.11.001

Hinds, J. y Sparks, P. (2009). Investigating Environmental Identity, Well-Being, and Meaning. Ecopsychology, 1(4), 181-186. DOI: https:// doi.org/10.1089/eco.2009.0026

Holland J. L. (1997). Making vocational choices: A theory of vocational personalities and work environments (3rd edition). Odessa, FL: Psychological Assessment Resources.

Howell, J. P. (2017). Waste governance and ecological identity in Maui, Hawaii, USA. Geoforum, 79, 81-89. DOI: https://doi.org/10.1016/j. geoforum.2016.12.015

Jaksha, A. P. y Johnson, B. (2013). Environmental identity: A new approach to understanding students' participation in environmental learning programs (tesis doctoral). University of Arizona Campus Repository.

Jia, F., Soucie, K., Alisat, S., Curtin, D. y Pratt, M. (2017). Are environmental issues moral issues? Moral identity in relation to protecting the natural world. Journal of Environmental Psychology, 52, 104-113. DOI: https://doi.org/10.1016/j.jenvp.2017.06.004

Kashima, Y., Paladino, A. y Margetts, E. A. (2014). Environmentalist identity and environmental striving. Journal of Environmental Psychology, 38, 64-75. DOI: https://doi.org/10.1016/j. jenvp.2013.12.014

Lawrence, E. K. (2012). Visitation to natural areas on campus and its relation to place identity and environmentally responsible behaviors. Journal of Environmental Education, 43(2), 93106. DOI: https://doi.org/10.1080/00958964. $\underline{2011.604654}$

Lewicka, M. (2008). Place attachment, place identity, and place memory: Restoring the forgotten city past. Journal of Environmental Psychology, 28(3), 209-231. DOI: https://doi.org/10.1016/j. jenvp.2008.02.001

Li, D. X., Kim, S., Lee, Y. K. y Griffin, M. (2016). Sustainable Environmental Development: The Moderating Role of Environmental Identity. Asian Journal of Social Psychology, 19(4), 298-310. DOI: https://doi.org/10.1111/ ajsp. 12146

Lipovetsky, G. (2000). La era del Vacío. Ensayos sobre el individualismo contemporáneo, Barcelona: Anagrama.

López-Facal, R. y Jiménez-Aleixandre, M. P. (2009). Identities, social representations and critical thinking. Cultural Studies of Science Education, 4(3), 689-695. DOI: https://doi. org/10.1007/s11422-008-9134-9

Mallett, R. K. y Melchiori, K. J. (2016). Creating a water-saver self-identity reduces water use in residence halls. Journal of Environmental Psychology, 47, 223-229. DOI: https://doi.org/10.1016/j.jenvp.2016.07.001 
Marcouyeux, A. y Fleury-Bahi, G. (2011). Place-identity in a school setting: Effects of the place image. Environment and Behavior, 43(3), 344-362. DOI: https://doi. org/10.1177/0013916509352964

Martínez-Ponce, I. (2015). Identidad ambiental: La construcción de un concepto a partir del análisis de la plataforma Pro-Río (tesis doctoral). Universidad de Alicante, España.

Meijers, F., Lengelle, R., Kopnina, H. (2016). Environmental Identity and Natural Resources: A Dialogical Learning Process. Resources, 5, 11. DOI: https://doi.org/10.3390/ resources5010011

Molina, A., Pérez, M., Castaño, N., Bustos, E., Suárez, O. y Sánchez, M. (2012). Mapeamiento informacional bibliográfico en el campo de la enseñanza de las ciencias, contexto y diversidad cultural: el caso del Journal Cultural Studies in Science Education (CSSE). Revista EDUCyT, volumen extra, 197-222.

Morton, T. A., van der Bles, A. M. y Haslam, S. A. (2017). Seeing our self-reflected in the world around us: The role of identity in making (natural) environments restorative. Journal of Environmental Psychology, 49, 65-77. DOI: https://doi.org/10.1016/j. jenvp.2016.11.002

Murtagh, N., Gatersleben, B. y Uzzell, D. (2012). Self-identity threat and resistance to change: Evidence from regular travel behaviour. Journal of Environmental Psychology, 32(4), 318-326. DOI: https://doi.org/10.1016/j. jenvp.2012.05.008

Navarro, O., Olivos, P. y Fleury-Bahi, G. (2017). "Connectedness to nature scale": Validity and reliability in the French context. Frontiers in Psychology, 8 (DEC), 1-8. DOI: https://doi. org/10.3389/fpsyg.2017.02180

Noguera, A. P. (2004). El reencantamiento del mundo. Colombia: Programa de las Naciones Unidas para el Medio Ambiente (PNUMA) - Universidad Nacional de Colombia, Manizales.
Olivos, P. y Aragonés, J. I. (2011). Propiedades psicométricas de la escala de identidad ambiental (EID). Psyecology, 2, 65-74. DOI: https:// doi.org/10.1174/217119711794394653

Olivos, P., Talayero, F., Aragonés, J. I. y Moyano-Díaz, E. (2014). Dimensiones del comportamiento proambiental y su relación con la conectividad e identidad ambientales. Dossiê: Psicologia Ambiental Comportamento Pró-Ambiental e Sustentabilidade, 45(3), 369-376.

Pavlova, M. K. y Silbereisen, R. K. (2015). Supportive Social Context and Intentions for Civic and Political Participation: An Application of the Theory of Planned Behaviour. Journal of Community y Applied Social Psychology, 25, 432-446. DOI: https://doi.org/10.1002/ casp. 2223

Perez, M. R y Porras, Y. (2005). La complejidad en el marco de una propuesta pluriparadigmática. Tecné, Episteme y Didaxis: TED, 17, 104-116. DOI: https://doi.org/10.17227/ted.num17-413

Plantin, C. (2014). Lengua, argumentación y aprendizajes escolares. Tecné, Episteme y Didaxis: TED, 36, 95-114. DOI: https://doi. org/10.17227/01213814.36ted95.114

Porras, Y., Pérez, M., Tuay, R., Alzate, M., Cuervo, F. y Roncancio, M. (2014). Retos y oportunidades de la Educación Ambiental en el siglo XXI. Bogotá: Fondo Editorial Universidad Pedagógica Nacional.

Quintana, A. y Montgomery, W. (2006). Psicología: tópicos de actualidad. Lima: UNMSM.

Riley, M. (2016). How Does Longer Term Participation in Agri-environment Schemes [re]Shape Farmers' Environmental Dispositions and Identities? Land Use Policy, 52, 62-75. DOI: https:// doi.org/10.1016/j.landusepol.2015.12.010

Robertson, D. (2013). Knowing Your Place: The Formation and Sustenance of Class-Based Place Identity. Housing, Theory and Society, 30(4), 368-383. DOI: https://doi.org/10.1080/14036 $\underline{096.2012 .755472}$

Rodríguez, M. P. y Ortega, W. (2017). Un mariposario como estrategia didáctica para desarrollar 
identidad ambiental en los estudiantes del grado 702 del Colegio Simón Bolívar de Suba (profundización). Seres, Saberes y Contextos, 2, 5-12.

Santos, B. S. (2009). Una epistemología del sur: la reinvención del conocimiento y la emancipación social. Buenos Aires: Clacso, Siglo $\mathrm{XXI}$.

Shaolian, D. (2017). Impacts of urban renewal on the place identity of local residents-a case study of Sunwenxilu traditional commercial street in Zhongshan City, Guangdong Province, China. Journal of Heritage Tourism, 12(3), 311-326. DOI: https://doi.org/10.1080/17438 73X.2016.1242591

Smart, J. C. y Thompson, M. D. (2001). The Environmental Identity Scale and Differentiation among Environmental Models in Holland's Theory. Journal of Vocational Behavior, 58(3), 436-452. DOI: https://doi.org/10.1006/ jvbe.2000.1760

Sola, E. (2014) Projeto Escola y Universidade: A formação do sujeto ecológico. Revista Brasileira de Educação Ambiental, 9(2), 436-450.

Spence, E., Pidgeon, N. y Pearson, P. (2018). UK public perceptions of Ocean Acidification The importance of place and environmental identity. Marine Policy, 97, 1-7. DOI: https:// doi.org/10.1016/j.marpol.2018.04.006

Stapleton, S. R. (2015). Environmental identity development through social interactions, action, and recognition. Journal of Environmental Education, 46(2), 94-113. DOI: https://doi.org/10. $\underline{1080 / 00958964.2014 .1000813}$

Stets, E. J. y Biga, F. C. (2003). Bringing Identity Theory into Environmental Sociology. Sociological Theory, 21(4), 398-423. DOI: https://doi. org/10.1046/j.1467-9558.2003.00196.x

Swim, J. K., Zawadzki, S. J., Cundiff, J. L. y Lord, B. (2014). Environmental identity and community support for the preservation of open space. Human, 20(2), 133-156. DOI: https://doi. org/10.22459/HER.20.02.2014.07
Thomashow, M. (1996). Ecological Identity: Becoming a Reflective Environmentalist. Cambridge, EE. UU.: MIT Press.

Tugurian, L. P. y Carrier, S. J. (2017). Children's environmental identity and the elementary science classroom. Journal of Environmental Education, 48(3), 143-153. DOI: https://doi.or g/10.1080/00958964.2016.1191415

Ujang, N. y Zakariya, K. (2015). The Notion of Place, Place Meaning and Identity in Urban Regeneration. Procedia-Social and Behavioral Sciences, 170, 709-717. DOI: https://doi.org/10.1016/j.sbspro.2015.01.073

Van der Werff, E., Steg, L. y Keizer, K. (2013). It is a moral issue: The relationship between environmental self-identity, obligation-based intrinsic motivation and pro-environmental behaviour. Global Environmental Change, 23(5), 1258-1265. DOI: https://doi.org/10.1177/0013916512475209

Van der Werff, E., Steg, L. y Keizer, K. (2014). I Am What I Am, by Looking Past the Present: The Influence of Biospheric Values and Past Behavior on Environmental Self-Identity. Environment and Behavior, 46(5), 626-657. DOI: https://doi. org/10.1177/0013916512475209

Varela, F. (1996) Ética y acción. Santiago de Chile: Dolmen Ediciones.

Wang, G., He, Q., Meng, X., Locatelli, G., Yu, T. y Yan, X. (2017). Exploring the impact of megaproject environmental responsibility on organizational citizenship behaviors for the environment: A social identity perspective. International Journal of Project Management, 35(7), 1402-1414. DOI: https://doi.org/10.1016/j. ijproman.2017.04.008

White, D. D., Virden, R. J. y Van Riper, C. J. (2008). Effects of place identity, place dependence and experience-use history on perceptions of recreation impacts in a natural setting. Environmental Management, 42(4), 647-657. DOI: https://doi.org/10.1007/ s00267-008-9143-1 
Winter, P. L. y Chavez, D. J. (2008). Wildland recreationists natural resource management purposes and preferences: a connection to environmental identity. En D. Chavez, P. Winter, y J. Absher (eds.), Recreation visitor research: studies of diversity. Gen Tech. Rep. PSW-GTR-210. Albany, EE. UU.: Department of Agriculture, Forest Service, Pacific Southwest Research Station.
Whitmarsh, L. y O'Neill, S. (2010). Green identity, green living? The role of pro-environmental self-identity in determining consistency across diverse pro-environmental behaviours. Journal of Environmental Psychology, 30(3), 305-314. DOI: https://doi.org/10.1016/j. jenvp.2010.01.003 